\title{
Periosteal osteosarcoma and Marfan's syndrome: A case report and literature review
}

\author{
GUO-PING XIE $^{1^{*}}$, HUI-JUAN SONG ${ }^{2 *}$, NAN JIANG ${ }^{1 *}$, CHENG-HE QIN ${ }^{1}$, \\ LEI WANG ${ }^{1}$, SHAO-YONG XU ${ }^{1}$ and BIN YU ${ }^{1,3}$ \\ ${ }^{1}$ Department of Orthopedics and Traumatology; ${ }^{2}$ Department of Nursing; \\ ${ }^{3}$ Key Laboratory of Bone and Cartilage Regenerative Medicine of Guangdong Province, \\ Nanfang Hospital, Southern Medical University, Guangzhou, Guangdong 510515, P.R. China
}

Received September 12, 2014; Accepted June 5, 2015

DOI: $10.3892 / \mathrm{ol} .2015 .3871$

\begin{abstract}
Periosteal osteosarcoma (POS) is a rare primary malignant bone tumor arising from the surface of long bones. In addition, Marfan's syndrome (MFS) is an infrequent hereditary autosomal dominant connective tissue disorder with high penetrance and variable phenotypes, which primarily affects the ocular, skeletal and cardiovascular systems. The present study reported a case of POS and MFS co-occurring in a child. A 6-year-old girl with MFS presented with pain, swelling and deformity in the right thigh following a fall. The patient was diagnosed with a right femoral shaft fracture and underwent open internal fixation surgery at a local hospital. At 2 weeks following surgery, the patient's parents observed increased swelling in the right thigh and thus, revisited the clinic. X-ray examination revealed extensive osteotylus around the fracture site and the clinician decided to remove the internal fixation. Following removal of the implant, aggravated swelling and superficial venous engorgement were observed. The patient was then admitted to Nanfang Hospital, where magnetic resonance imaging was performed, which identified symptoms of an abnormal periosteal reaction with bone erosion, indicating POS. The patient underwent a wide resection of the tumor and the histopathological examination confirmed the diagnosis of POS. No recurrence was identified at 9 months postoperatively. In conclusion, the present case report may result in increased awareness of the possibility of malignant bone tumors in a hereditary patient with osteotylus overgrowth following fracture surgery; in addition, the present case indicated a possible correlation between POS and MFS.
\end{abstract}

Correspondence to: Dr Bin Yu, Department of Orthopedics and Traumatology, Nanfang Hospital, Southern Medical University, 1838 Guangzhou Avenue North, Guangzhou, Guangdong 510515, P.R. China

E-mail: nanfanghot@126.com

*Contributed equally

Key words: periosteal osteosarcoma, Marfan's syndrome, femoral shaft fracture

\section{Introduction}

Osteosarcoma is a frequent primary malignant bone tumor with predilection in children and adolescents (1), the oncogenesis of which has been reported to be partially associated with transforming growth factor $\beta$ (TGF- $\beta$ ) (2). Periosteal osteosarcoma (POS), an intermediate-grade chondroblastic osteosarcoma arising from the surface of the long bones, accounts for $<2 \%$ of all osteosarcomas $(3,4)$. Previous studies have demonstrated that certain hereditary diseases including Rothmund-Thomson syndrome, Bloom syndrome and Li-Fraumeni syndrome may increase the risk of osteosarcoma $(5,6)$.

Marfan's syndrome (MFS), a rare autosomal dominant hereditary disorder of connective tissue, primarily affects the ocular, skeletal and cardiovascular systems, with high penetrance and variable phenotypes (7). MFS was reported to be associated with perturbations in TGF- $\beta$ biology, which were commonly found to result from mutations in the fibrillin-1 gene $(8,9)$. One previous study reported the simultaneous occurrence of MFS and osteosarcoma of the foot in a patient (10). However, it remains to be elucidated whether POS is associated with MFS.

The present study reported a case of POS and MSF co-occurring in a 6-year-old girl, the former of which induced a pathological femoral shaft fracture. The present report described the process of the disorders and reviewed the relevant literature.

\section{Case report}

Overview. A 6-year-old girl visited the clinic of Nanfang Hospital, Southern Medical University (Guangzhou, China) with aggravating swelling in the right thigh following two previous surgeries of internal fixation and implant removal, which were performed due to a femoral shaft fracture in her right leg. The present study was approved by medical ethics committee of Nanfang Hospital. Written informed consent from the patient's family was provided, and the patients' records were anonymized and de-identified prior to analysis.

Medical history. Abnormal symptoms were denied prior to the presentation of the fracture in the patient. A blood relationship between the patient's parents was also denied. The patient's 


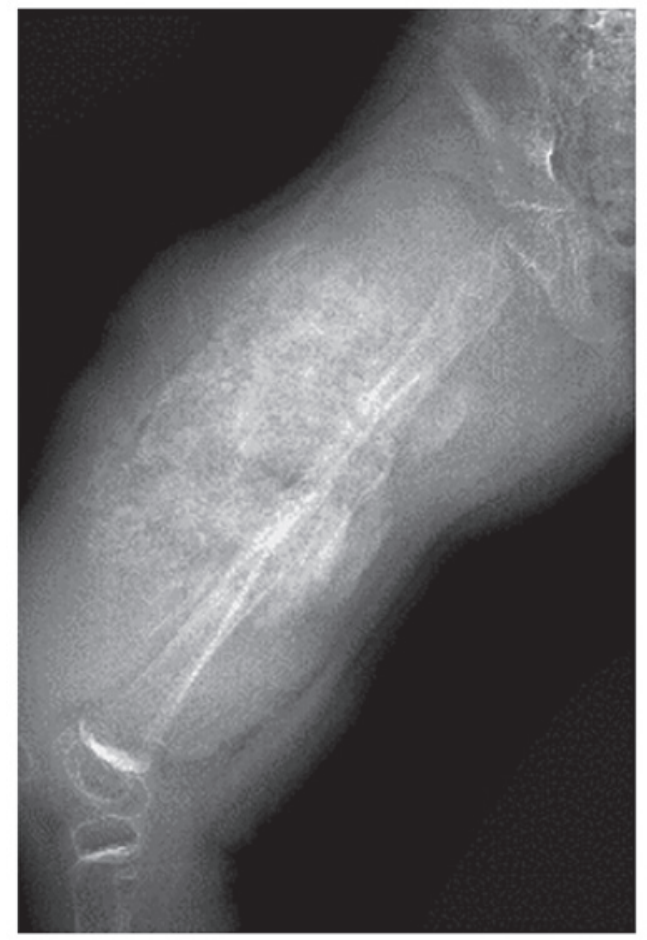

Figure 1. X-ray image of the patient. Preoperative X-ray demonstrating extensive osteolytic destruction, asymmetrical periosteal reaction with Codman's triangle and cortex thickening in the femoral shaft.

father had previously received a diagnosis of MFS; however, heredity disorders were not present in other members of the family.

Initial presentation. Subsequent to a fall during exercise, the patient presented with pain, swelling, deformity and disability in the right thigh. Radiograph images performed at a local hospital revealed a single fracture in the right femoral shaft. The patient was admitted and received fracture fixation surgery a week later. Symptomatic treatment and nutritional support were administered postoperatively. At 2 weeks post surgery, aggravated swelling was observed and the patient returned to the local hospital. X-ray examination revealed the formation of an extensive osteotylus in the affected site. The patient was readmitted and underwent removal of the implant 1 month following the initial surgery. Aggravated swelling appeared again, accompanied with superficial venous engorgement following the second surgery. The patient was then brought to Nanfang Hospital.

Medical examination. The patient was $123 \mathrm{~cm}$ in height and $24 \mathrm{~kg}$ in weight. Physical examination revealed a normal spinal curvature and a long slender neck. The upper to lower segment ratio was 0.82 . Joint hypermobility was identified in the patient's hands and fingers. Optic examination showed a slight impairment in both of the eyes. The circumference at $10 \mathrm{~cm}$ above patella of the right thigh was $52 \mathrm{~cm}$, compared with $31 \mathrm{~cm}$ in the left thigh. In addition, tension blisters as well as superficial venous engorgement were observed. However, the sensation of the affected limb was normal.

Laboratory results demonstrated significantly increased serum levels of white blood cells $\left(28.73 \times 10^{9} / 1\right.$; normal range, $\left.3.5-9.5 \times 10^{9} / 1\right)$, C-reactive protein $(81.1 \mathrm{mg} / 1$; normal range, $0-5 \mathrm{mg} / \mathrm{l}$ ) and alkaline phosphatase (976.4-1100 U/l; normal range, 50-400 U/l). However, the serum erythrocyte sedimentation rate $(21 \mathrm{~mm} / \mathrm{l})$ was only slightly elevated compared with the upper value of normal range $(20 \mathrm{~mm} / \mathrm{l})$. Radiograph images revealed extensive osteolytic destruction, asymmetrical periosteal reaction with Codman's triangle and cortex thickening, which indicated the possibility of osteosarcoma. No X-ray abnormities were identified in the hip or the knee (Fig. 1), nor in the chest. Magnetic resonance imaging (MRI) of right thigh revealed abnormal signals in $\mathrm{T}_{1}$ and $\mathrm{T}_{2}$-weighted images (Fig. 2A-C). Spinal MRI revealed a biconcave sign in the partial vertebral bodies (Fig. 2D). An electrocardiogram demonstrated normal sinus rhythms without other types of arrhythmia. In addition, an echocardiograph revealed normal sizes and motions of the ventricles, atria and valves. MFS was diagnosed according to the aforementioned clinical features and the associated family history.

Operative procedure and postoperative follow up. Osteosarcoma was suspected, based on the following factors: i) Extensive osteotylus was observed $<1$ month after fracture; ii) extensive osteolytic destruction, asymmetrical periosteal reaction with Codman's triangle and cortex thickening were observed (Fig. 1); and iii) clinical symptoms and laboratory tests also indicated abnormalities, including tension blisters and superficial venous engorgement, as well as signifiantly increased serum levels of alkaline phosphatase, white blood cells and C-reactive protein. Thus, radical resection of the tumor was proposed according to the limb salvage requirement of the patient as well as the patient's parents. Surgery was performed under general anesthesia. During surgery, the muscles around the femur were observed to have a fatty-like change and the aberrant bone grew widely around the bone shaft. The patient underwent thorough resection of the tumor and reconstruction. Resected tissues underwent histopathological and immunohistochemical examinations (Fig. 3A and B). Histopathological examination revealed marked chondroid differentiation with hypercellularity and prominent nuclear pleomorphism. Immunohistochemical examination revealed positive expression of CD99 (+), osteopontin (+, weakly), Ki-67 (+, 50\%), p53 (+, individually) and S-100 (+), as well as negative expression of B cell lymphoma 2 (-). Outcomes of the above examinations confirmed the diagnosis of POS. Postoperative radiographs demonstrated that the primary tumor was resected completely and the intramedullary fixation of the femur was in place (Fig. 4).

\section{Discussion}

Osteosarcoma is one of the most frequent types of primary malignant bone tumors, accounting for $\sim 15 \%$ of all bone tumors (11). In addition, the incidence rate of osteosarcoma arising from the surface of the long bones is markedly lower compared with those from other sites of the bone. Surface osteosarcomas are classified into three main types, including parosteal (juxtacortical), periosteal and high-grade osteosarcoma (12). POS was first reported by Ewing (13) in 1939; subsequently, in 1976, Unni et al (14) described the characteristics of POS pathology based on 23 patients. As 

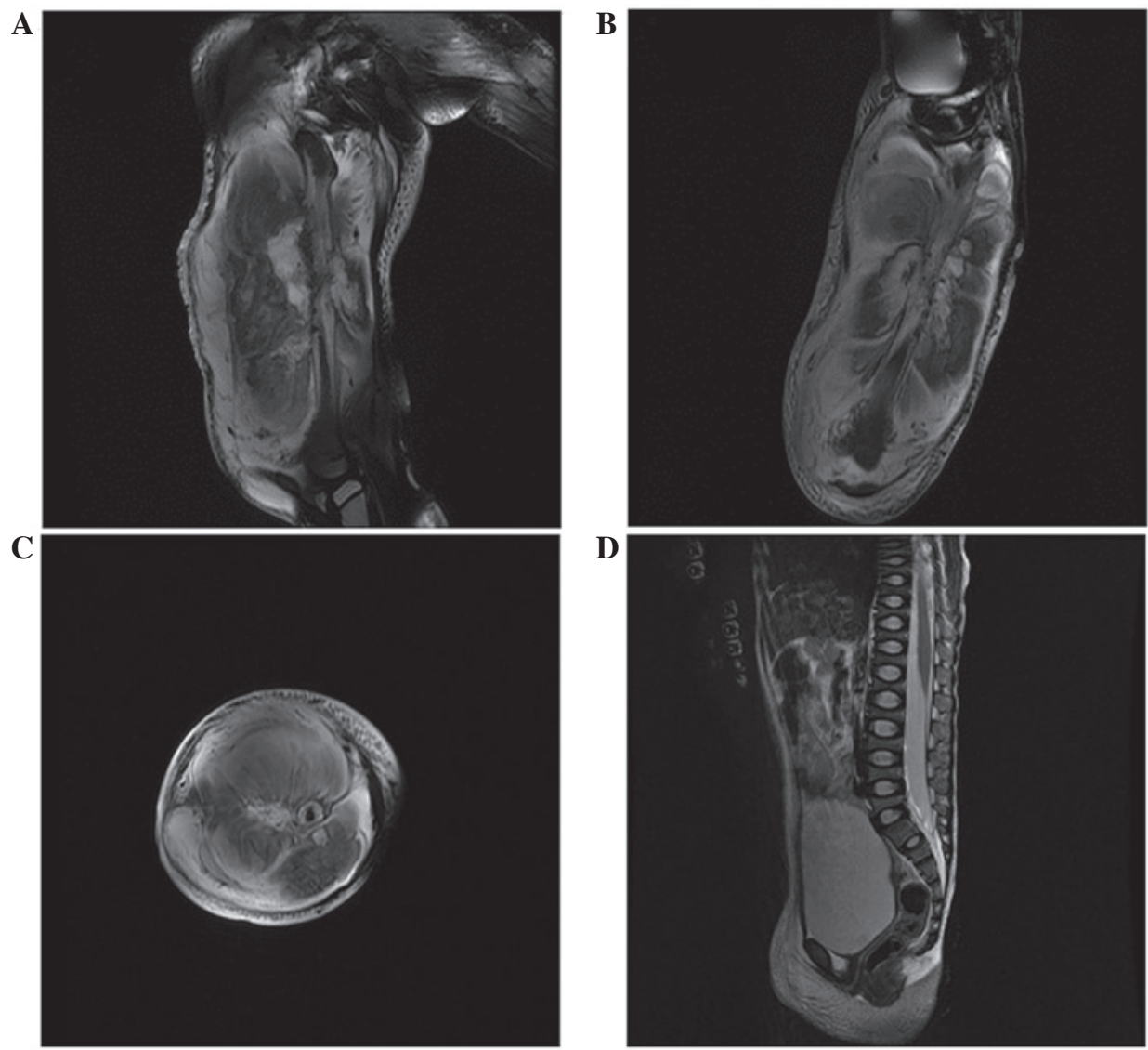

Figure 2. MRI scans of the patient. (A-C) MRI of the right thigh showed heterogeneous high signal intensity on $\mathrm{T}_{2}$-weighted scans. Intermediate signal intensity on $\mathrm{T}_{1}$-weighted scans was also observed (not shown). (D) Spinal MRI revealed biconcave sign in the partial vertebral bodies. MRI, magnetic resonance imaging.
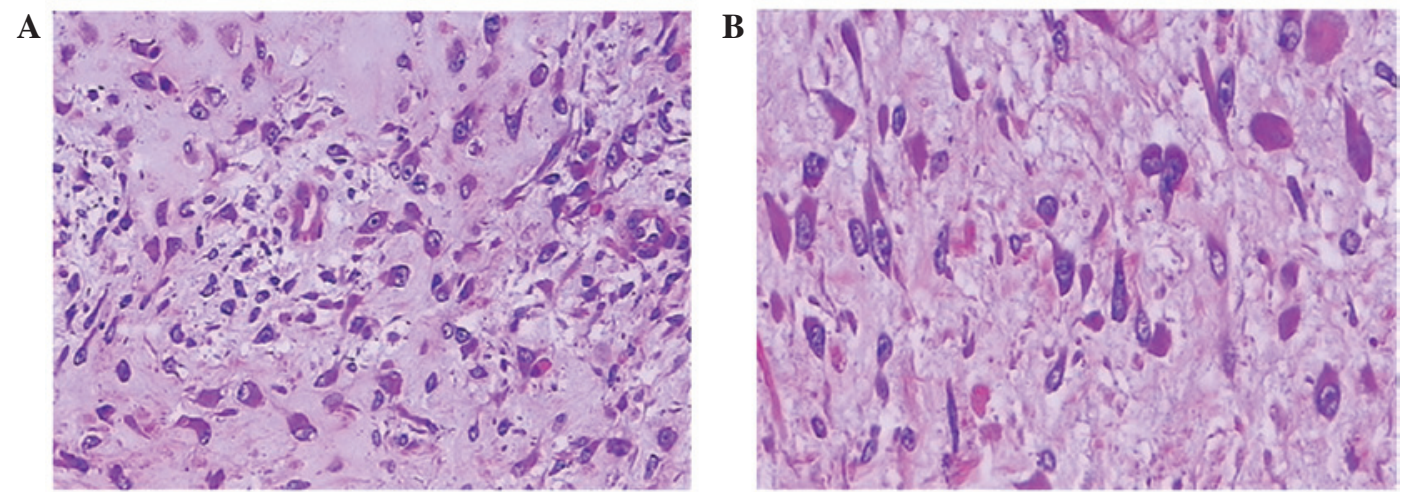

Figure 3. Histopathological examination of the resected tumor. Histopathological images of the resected lesion at (A) x10 magnification and (B) x40 magnification demonstrated notable chondroid differentiation with hypercellularity and prominent nuclear pleomorphism.

a type of intermediate-grade osteosarcoma, POS predominantly occurs in males and patients in the second decade of life $(15,16)$. POS usually arises from the diaphysis or the meta-diaphyseal surface of the tibia and femur; in addition, POS is less aggressive and has a better prognosis compared with other frequent types of osteosarcoma $(17,18)$. However, controversy still surrounds the treatment of this disease. The effect of chemotherapy treatment on the prognosis of POS patients remains indefinite (15), although it often demonstrates a promising clinical efficacy in the treatment of osteosarcoma. Grimer et al (15) reported the good prognosis of POS patients following only resection of the tumor. Thus, the case in the present study only received resection of the tumor without chemotherapy or radiotherapy. The role of the two adjunctive therapy methods in the treatment of POS requires further investigation.

MFS was first described by Antoine-Bernard Marfan in 1898. MFS is a rare autosomal dominantly inherited systemic connective tissue disorder with an estimated prevalence of $1-3$ per $10,000(19,20)$. The disease primarily affects the cardiovascular, skeletal and ocular systems, with skin, fascia, lung and adipose tissue occasionally involved (21). Diet et al (22) indicated that MFS was associated with the genetic defects in the chromosome loci $\mathrm{D}_{15} \mathrm{~S}_{25}$ and $\mathrm{D}_{15} \mathrm{~S}_{1}$. The 


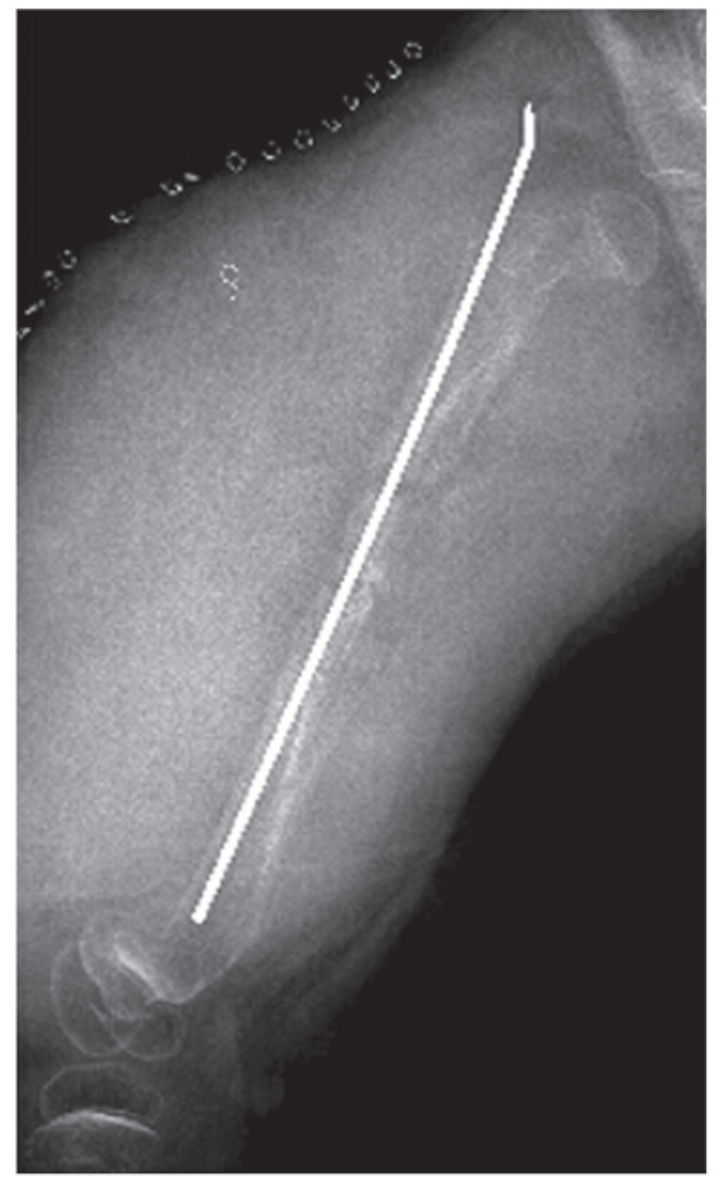

Figure 4. Postoperative X-rays confirming the complete resection of the primary tumor and the placement of the intramedullary fixation.

disease exhibited a high penetrance and a marked inter- and intrafamilial variability (23); however, there remains to be a lack of effective methods for the treatment of this disorder. Cardiovascular deformities are the primary fatal factor of MFS; thus, the prevention of life-threatening cardiovascular complications with MFS is vital. The main strategies for this include lifestyle modifications, regular echocardiographic assessment, pharmacological treatment and prophylactic surgery (24). In the present study, the patient did not present with any abnormalities of the cardiovascular system.

To the best of our knowledge, only one previous study has reported a case of simultaneous osteosarcoma and MFS (10); in this previous case, the osteosarcoma was located in the foot. Compared with the previous case, the present case had several different characteristics. Initially, the affected site was the femur shaft. Additionally, fracture was the main reason that accounted for the patient's visit to the clinic of a local hospital, which treated the patient as a simple femoral shaft fracture. Subsequently, it was revealed the patient suffered from the co-occurrence of MFS and POS. The simultaneous occurrence of these two rare disorders in a patient may indicate a correlation between MSF and POS. It was previously reported that heterozygous mutations in TGF- $\beta$ receptor 2 were associated with several malignancies and genetic connective-tissue disorders (25). Previous studies also indicated that TGF- $\beta$ had a crucial role in the aortopathy of MFS (26-28). In addition, one study suggested that TGF- $\beta$ was an autocrine factor that regulated the growth of human osteosarcomas (2). Therefore, it may be inferred that TGF- $\beta$ is a potential connection between the occurrence of MSF and POS.

In conclusion, to the best of our knowledge, the present study was the first to report a case of simultaneous POS and MFS in a child. The case report identified the characteristics of POS and key points of the disease in the process of diagnosis and treatment. The present study may therefore increase the awareness of orthopedists on the possibility of malignant bone tumor in a hereditary patient with osteotylus overgrowth following fracture surgery. In addition, due to the simultaneous appearance of POS and MFS in the present case, it was suggested that further studies should be conducted to determine whether there is an association between the two rare disorders.

\section{Acknowledgements}

The authors thank Professor Allen P. Liang for revising and editing this manuscript.

\section{References}

1. Durnali A, Alkis N, Cangur S, Yukruk FA, Inal A, Tokluoglu S, Seker MM, Bal O, Akman T, Inanc M, et al: Prognostic factors for teenage and adult patients with high-grade osteosarcoma: An analysis of 240 patients. Med Oncol 30: 624, 2013.

2. Franchi A, Arganini L, Baroni G, Calzolari A, Capanna R, Campanacci D, Caldora P, Masi L, Brandi ML and Zampi G: Expression of transforming growth factor beta isoforms in osteosarcoma variants: Association of TGF beta 1 with high-grade osteosarcomas. J Pathol 185: 284-289, 1998.

3. Cesari M, Alberghini M, Vanel D, Palmerini E, Staals EL, Longhi A, Abate M, Ferrari C, Balladelli A and Ferrari S: Periosteal osteosarcoma: A single-institution experience. Cancer 117: 1731-1735, 2011.

4. Maheshwari AV, Jelinek JS, Seibel NL, Meloni-Ehrig AM, Kumar D and Henshaw RM: Bilateral synchronous tibial periosteal osteosarcoma with familial incidence. Skeletal Radiol 41: 1005-1009, 2012.

5. Fuchs B and Pritchard DJ: Etiology of osteosarcoma. Clin Orthop Relat Res: 40-52, 2002.

6. Malkin D, Li FP, Strong LC, Fraumeni JF Jr, Nelson CE, Kim DH, Kassel J, Gryka MA, Bischoff FZ and Tainsky MA: Germ line p53 mutations in a familial syndrome of breast cancer, sarcomas and other neoplasms. Science 250: 1233-1238, 1990.

7. Summers KM, Xu D, West JA, et al: An integrated approach to management of Marfan syndrome caused by an FBN1 exon 18 mutation in an Australian Aboriginal family. Clin Genet 65: 66-69, 2004.

8. Pearson GD, Devereux R, Loeys B, et al: Report of the national heart, lung and blood institute and national marfan foundation working group on research in Marfan syndrome and related disorders. Circulation 118: 785-791, 2008.

9. Dietz HC, Cutting GR, Pyeritz RE, Maslen CL, Sakai LY, Corson GM, Puffenberger EG, Hamosh A, Nanthakumar EJ and Curristin SM: Marfan syndrome caused by a recurrent de novo missense mutation in the fibrillin gene. Nature 352: 337-339, 1991.

10. Roopnariane A, Freed RJ, Price S, Fox EJ and Ritty TM: Osteosarcoma in a Marfan patient with a novel premature termination codon in the FBN1 gene. Connect Tissue Res 52: 157-165, 2011.

11. Murphey MD, Robbin MR, McRae GA, Flemming DJ, Temple HT and Kransdorf MJ: The many faces of osteosarcoma. Radiographics 17: 1205-1231, 1997.

12. Schajowicz F, McGuire MH, Santini Araujo E, Muscolo DL and Gitelis S: Osteosarcomas arising on the surfaces of long bones. J Bone Joint Surg Am 70: 555-564, 1988.

13. Ewing J: A review of the classification of bone tumours. Bull Am Coll Surg 24: 290-295, 1939.

14. Unni KK, Dahlin DC and Beabout JW: Periosteal osteogenic sarcoma. Cancer 37: 2476-2485, 1976. 
15. Grimer RJ,Bielack S,Flege S, Cannon SR, Foleras G, Andreeff I, Sokolov T, Taminiau A, Dominkus M, San-Julian M, et al: Periosteal osteosarcoma-a European review of outcome. Eur J Cancer 41: 2806-2811, 2005.

16. Ritts GD, Pritchard DJ, Unni KK, Beabout JW and Eckardt JJ: Periosteal osteosarcoma. Clin Orthop Relat Res 219 299-307, 1987.

17. Hall RB, Robinson LH, Malawar MM and Dunham WK: Periosteal osteosarcoma. Cancer 55: 165-171, 1985.

18. Lim C, Lee H, Schatz J, Alvaro F, Boyle R and Bonar SF: Case report: Periosteal osteosarcoma of the clavicle. Skeletal Radiol 41: 1011-1015, 2012.

19. Gray JR, Bridges AB, Faed MJ, Pringle T, Baines P, Dean J and Boxer M: Ascertainment and severity of Marfan syndrome in a Scottish population. J Med Genet 31: 51-54, 1994.

20. Judge DP and Dietz HC: Marfan's syndrome. Lancet 366: 1965-1976, 2005.

21. Loeys BL, Dietz HC, Braverman AC, Callewaert BL, De Backer J, Devereux RB, Hilhorst-Hofstee Y, Jondeau G, Faivre L, Milewicz DM, et al: The revised Ghent nosology for the Marfan syndrome. J Med Genet. 47: 476-485, 2010.

22. Dietz HC, Pyeritz RE, Hall BD, Cadle RG, Hamosh A, Schwartz J, Meyers DA and Francomano CA: The Marfan syndrome locus: Confirmation of assignment to chromosome 15 and identification of tightly linked markers at 15q15-q21.3. Genomics 9: 355-361, 1991.
23. Pyeritz RE: The Marfan syndrome. Annu Rev Med 51: 481-510, 2000.

24. Cook JR and Ramirez F: Clinical, diagnostic and therapeutic aspects of the Marfan syndrome. Adv Exp Med Biol 802: 77-94, 2014.

25. Mizuguchi T, Collod-Beroud G, Akiyama T, Abifadel M, Harada N, Morisaki T, Allard D, Varret M, Claustres M, Morisaki H, et al: Heterozygous TGFBR2 mutations in Marfan syndrome. Nat Genet 36: 855-860, 2004.

26. Mizuguchi $\mathrm{T}$ and Matsumoto $\mathrm{N}$ : Recent progress in genetics of Marfan syndrome and Marfan-associated disorders. J Hum Genet 52: 1-12,2007.

27. Sawaki D and Suzuki T: Targeting transforming growth factor- $\beta$ signaling in aortopathies in Marfan syndrome. Circ J 77: 898-899, 2013.

28. Agg B, Benke K, Szilveszter B, Pólos M, Daróczi L, Odler B, Nagy ZB, Tarr F, Merkely B and Szabolcs Z: Possible extracardiac predictors of aortic dissection in Marfan syndrome. BMC Cardiovasc Disord 14: 47, 2014. 